University of Nebraska - Lincoln

DigitalCommons@University of Nebraska - Lincoln

$11-2013$

\title{
Carrier-envelope-phase-induced asymmetries in double ionization of helium by an intense few-cycle XUV pulse
}

Jean Marcel Ngoko Djiokap

University of Nebraska-Lincoln, marcelngoko@unl.edu

N. L. Manakov

Voronezh State University, Russia, manakov@phys.vsu.ru

A. V. Meremianin

Voronezh State University, meremianin@phys.vsu.ru

Anthony F. Starace

University of Nebraska-Lincoln, astarace1@unl.edu

Follow this and additional works at: https://digitalcommons.unl.edu/physicsstarace

Part of the Atomic, Molecular and Optical Physics Commons, Elementary Particles and Fields and String Theory Commons, and the Plasma and Beam Physics Commons

Ngoko Djiokap, Jean Marcel; Manakov, N. L.; Meremianin, A. V.; and Starace, Anthony F., "Carrier-envelopephase-induced asymmetries in double ionization of helium by an intense few-cycle XUV pulse" (2013). Anthony F. Starace Publications. 201.

https://digitalcommons.unl.edu/physicsstarace/201

This Article is brought to you for free and open access by the Research Papers in Physics and Astronomy at DigitalCommons@University of Nebraska - Lincoln. It has been accepted for inclusion in Anthony F. Starace Publications by an authorized administrator of DigitalCommons@University of Nebraska - Lincoln. 


\title{
Carrier-envelope-phase-induced asymmetries in double ionization of helium by an intense few-cycle XUV pulse
}

\author{
J. M. Ngoko Djiokap, ${ }^{1}$ N. L. Manakov, ${ }^{2}$ A. V. Meremianin, ${ }^{2}$ and Anthony F. Starace ${ }^{1}$ \\ ${ }^{1}$ Department of Physics and Astronomy, University of Nebraska, Lincoln, Nebraska 68588-0299, USA \\ ${ }^{2}$ Department of Physics, Voronezh State University, Voronezh 394006, Russia
}

(Received 29 May 2013; revised manuscript received 22 October 2013; published 13 November 2013)

\begin{abstract}
The carrier-envelope-phase (CEP) dependence of electron angular distributions in double ionization of He by an arbitrarily polarized, few-cycle, intense XUV pulse is formulated using perturbation theory (PT) in the pulse amplitude. Owing to the broad pulse bandwidth, interference of first- and second-order PT amplitudes produces asymmetric angular distributions sensitive to the CEP. The PT parametrization is shown to be valid by comparing with results of solutions of the full-dimensional, two-electron time-dependent Schrödinger equation for the case of linear polarization.
\end{abstract}

DOI: 10.1103/PhysRevA.88.053411

PACS number(s): $32.80 . \mathrm{Fb}, 02.70 . \mathrm{Dh}, 02.70 . \mathrm{Hm}, 32.80 . \mathrm{Rm}$

\section{INTRODUCTION}

Theoretical analyses and experimental measurements of the differential cross sections for single-photon, double photoionization (DPI) of the He atom have a long history of providing valuable insights into this most fundamental interacting threebody Coulomb problem [1]. In particular, angular momentum, parity, and identical particle exchange considerations have led to discovery of numerous rigorous selection rules for the finalstate electron angular distributions (ADs) [1,2]. Moreover, such analyses allow the $\mathrm{AD}$ to be parametrized in terms of dynamical and geometrical factors $[1,3,4]$.

Significant control of this fundamental process can be achieved by measuring the $\mathrm{AD}$ of an electron pair [with momentum directions $\hat{\mathbf{p}}_{1}$ and $\hat{\mathbf{p}}_{2}$ and energy $E=\left(p_{1}^{2}+\right.$ $\left.\left.p_{2}^{2}\right) /(2 m)\right]$ resulting from DPI of He by an intense, few-cycle XUV pulse with stabilized carrier-envelope phase (CEP) $\phi$. Indeed, for processes initiated by a pulse field, unlike those initiated by a monochromatic one, the physical observables are not invariant with respect to the sign of the shortpulse electric field vector $\mathbf{F}(t)$ or, equivalently, its generally complex polarization vector $\mathbf{e}\left(\mathbf{e} \cdot \mathbf{e}^{*}=1\right)$. Thus, changing $\phi \rightarrow(\phi \pm \pi)$, or $\mathbf{e} \rightarrow-\mathbf{e}$, "inverts" the temporal distribution of the pulse field $\mathbf{F}(t)$. Since the triply differential probability (TDP) for DPI, $d^{3} W / d E d \Omega_{\hat{\mathbf{p}}_{1}} d \Omega_{\hat{\mathbf{p}}_{2}} \equiv \mathcal{W}\left(\mathbf{p}_{1}, \mathbf{p}_{2}, \mathbf{e}\right)$ is a true scalar, the change $\mathbf{e} \rightarrow-\mathbf{e}$ is equivalent to similar changes of other vectors of the problem, i.e., $\left(\mathbf{p}_{1}, \mathbf{p}_{2}\right) \rightarrow\left(-\mathbf{p}_{1},-\mathbf{p}_{2}\right)$. Thus, the CEP induces an asymmetry in the $\mathrm{AD}$,

$$
\Delta \mathcal{W}=\mathcal{W}\left(\mathbf{p}_{1}, \mathbf{p}_{2}, \mathbf{e}\right)-\mathcal{W}\left(-\mathbf{p}_{1},-\mathbf{p}_{2}, \mathbf{e}\right),
$$

that vanishes after phase averaging and provides a prominent manifestation of CEP effects in short-pulse DPI.

Although the phenomenological result (1) is very general and valid for both perturbative and nonperturbative [in the pulse field $\mathbf{F}(t)$ ] regimes, the physical mechanisms responsible for the AD asymmetry, as well as the information on atomic dynamics provided by measuring this asymmetry, differ. Specifically, the infrared lasers used in strong-field physics have small photon energies and the laser-field coupling with the atom must be treated nonperturbatively when analyzing CEP-induced AD asymmetries in single ionization of an atom [5-7]. Moreover, it is well known that the $\mathrm{AD}$ asymmetry in the strong-field regime stems from interplay of closed classical electron trajectories originating from neighboring optical half cycles of a strong laser pulse field $[7,8]$. Hence the numerous rigorous selection rules derived for electron ADs resulting from DPI of an atom by one photon have very limited applicability in strong-field physics. Consequently, although strong-field experiments on DPI have demonstrated the sensitivity of the results to the CEP of a short laser pulse (see, e.g., Ref. [9]) and have gleaned information on electron dynamics from an analysis of the DPI angular distributions (see, e.g., Ref. [10]), analytic interpretations of such experiments are not at present available.

In contrast, owing to the high carrier frequency of XUV pulses, DPI by a few-cycle XUV pulse can be described using perturbation theory (PT) in the pulse field $\mathbf{F}(t)$. For single ionization of an atom by a few-cycle XUV pulse, a PT analysis [11] provides an ab initio parametrization of the ionized electron $\mathrm{AD}$ in terms of the key parameters of the process: the CEP of the pulse, the pulse polarization, and the electron momentum direction. This parametrization [11] for XUV pulse photoionization of He describes accurately numerical results obtained by solving the time-dependent Schrödinger equation (TDSE) [12-14]. In general in the PT regime, the $\mathrm{AD}$ asymmetry originates from interference of first- and second-order PT transition amplitudes to continuum states with the same energy $E$ but different parities [11]. [Note that for a long $(\approx$ monochromatic) pulse such interference is forbidden owing to dipole selection rules.] In DPI of $\mathrm{He}$, the magnitude of the $\mathrm{AD}$ asymmetry should thus be sensitive to interference of opposite-parity two-electron continuum states. In order that such interference effects can be observed, two conditions are necessary. First, the peak intensity of the XUV pulse must be high enough so that the second-order PT DPI amplitude has a magnitude that allows for significant interference with the first-order PT DPI amplitude. Numerical simulations have shown that XUV pulse intensities of order $1 \mathrm{PW} / \mathrm{cm}^{2}$ are necessary in order that the contribution of the second-order PT transition amplitude is significant $[11,12]$. Second, the few-cycle XUV pulses must have a sufficiently large bandwidth $\Delta \omega$ so that the electron energy spectra produced by the first- and second-order PT amplitudes overlap in energy. Where such overlap may occur can be estimated as in the following example. Consider the first- and second-order 
PT amplitudes for DPI by absorption of respectively one and two photons. The energy range above the He ground state for which the first-order PT amplitude is significant is $\omega \pm \Delta \omega$, while the corresponding energy range for the second-order PT amplitude is $2 \omega \pm 2 \Delta \omega$. Defining the high-energy position of the first-order PT amplitude by $\omega_{1}=\omega+\Delta \omega$ and the low-energy position of the second-order PT amplitude by $\omega_{2}=2 \omega-2 \Delta \omega$, one expects the energy location at which maximum interference of these first- and second-order amplitudes occurs to be in the vicinity of $\omega_{a v}=\left(\omega_{1}+\omega_{2}\right) / 2$. Experimentally, few-cycle XUV attosecond pulses with stable CEPs have been achieved $[15,16]$. Although attosecond pulse intensities at present are low, progress on increasing the intensities is being made (see, e.g., Refs. [17-24]).

Note that two-photon DPI of He has become almost a subfield unto itself owing to recent experiments involving XUV pulses produced by high-order harmonics [25] or by free-electron lasers [26-29] that observe this nonlinear process. Theoretically, there have been a large number of investigations of two-photon DPI of $\mathrm{He}$ involving short XUV pulses (see, e.g., Refs. [30-46]). Nearly all of these references provide information on pulse duration effects. However, only a few of these simulations involve few-cycle single XUV pulses [30,32,37,38,41,45]. Moreover, several of these $[30,32,38,41]$ indicate that the final two-electron wave packet is projected onto states with total angular momenta $L=0,2$ so that effects of the interference with neighboring odd-order PT amplitudes (i.e., the first order with $L=1$ and/or the third order with $L=1,3$ ) cannot be observed. Finally, all of these prior investigations of two-photon DPI of He are for the case of a linearly polarized XUV pulse and none of the few-cycle investigations include an analysis of CEP effects.

In this paper we present a parametrization of the nonlinear attosecond process of DPI of He by a few-cycle XUV pulse. The result is given in terms of the CEP $\phi$ of the pulse and the key vectors of the problem: the complex pulse polarization $\mathbf{e}$, and the electron momentum directions $\hat{\mathbf{p}}_{1}$ and $\hat{\mathbf{p}}_{2}$. Analysis of DPI by a few-cycle XUV pulse permits one to probe the $S$-, $P$-, and $D$-wave two-electron continua, whereas only the $P$ wave is accessible in ordinary DPI [1]. In contrast to single ionization of He treated in Ref. [11], the additional final-state electron momentum vector in DPI gives a more complex parametrization. We focus on the CEP dependence of the $\mathrm{AD}$ asymmetries for an arbitrarily polarized pulse. The parametrized AD is compared with solutions of the twoelectron TDSE for a linearly polarized pulse. The predicted asymmetries concern more than one particle, as the second electron is observed to behave differently depending on the orientation of the first electron with respect to the electric field. In this regard, we emphasize that our analytical analysis employs PT only in the XUV pulse interaction with the atom; the dynamic amplitudes include electron correlation effects to all orders. Moreover, our numerical simulations of DPI of He involve the full-dimensional solution of the two-electron time-dependent Schrödinger equation for the case of a linearly polarized XUV pulse, so that all electron correlation effects and interactions of the He atom with the XUV pulse are accounted for nonperturbatively and exactly to within our numerical accuracy.

\section{THEORETICAL ANALYSIS}

We consider the interaction of an atom with a shortpulse electric field $\mathbf{F}(t)=F_{0}(t) \operatorname{Re}\left[\mathbf{e} e^{-i(\omega t+\phi)}\right]$, where $F_{0}(t)$ is a smooth envelope function. It is convenient to define the Fourier transform of $\mathbf{F}(t): \hat{\mathbf{F}}(\epsilon)=\int_{-\infty}^{\infty} \mathbf{F}(t) e^{i \epsilon t} d t=$ $\mathbf{e}_{\phi} \hat{F}^{(+)}(\epsilon)+\mathbf{e}_{\phi}^{*} \hat{F}^{(-)}(\epsilon)$, where $\mathbf{e}_{\phi} \equiv \mathbf{e} e^{-i \phi}$ and $F^{( \pm)}(\epsilon) \equiv$ $\int_{-\infty}^{\infty} F_{0}(t) e^{i(\epsilon \mp \omega) t} d t / 2$. Within PT in the dipole interaction of an atom with a pulse electric field $\mathbf{F}(t)$, the amplitude $A$ for short-pulse DPI of an initial two-electron bound ${ }^{1} S_{0}$ state with energy $E_{0}$ may be written as $A=A_{1}+A_{2}$, where $A_{1}$ is the first-order PT amplitude for single-photon DPI to the continuum $P$ state of the ionized electron pair with energy $E$ and $A_{2}$ is the second-order PT amplitude for a transition (involving two photons) to the continuum with the same energy $E$ but with total angular momentum $L=0,2$. The DPI probability is thus proportional to $|A|^{2}=$ $\left|A_{1}\right|^{2}+2 \operatorname{Re}\left(A_{1}^{*} A_{2}\right)+\left|A_{2}\right|^{2}$. Owing to their different parities, the interference of the amplitudes $A_{1}$ and $A_{2}$, described by $2 \operatorname{Re}\left(A_{1}^{*} A_{2}\right)$, leads to asymmetries in the $\mathrm{ADs}$ of the two electrons. Moreover, as we shall show, these asymmetries may be controlled by the pulse CEP $\phi$. We neglect spin-orbit interactions, so that both amplitudes are scalars independent of the quantization axis. We adopt the same assumptions as in Ref. [11] (which are appropriate for a pulse bandwidth that is not too large): (i) the ionization amplitude $A_{1}$ is assumed to be dominated by the positive frequency term $\left(\hat{F}^{(+)}\right)$(describing photon absorption); (ii) the terms involving two successive negative frequency components $\left(\hat{F}^{(-)}\right)$(describing photon emission) in the second-order PT amplitude $A_{2}$ are neglected; (iii) $\left|A_{2}\right|^{2}$ is assumed to be small (except when $A_{1}=0$ ). For any geometry and polarization, the TDP for DPI thus equals

$$
\mathcal{W}\left(\mathbf{p}_{1}, \mathbf{p}_{2}, \mathbf{e}\right) \approx \mathcal{C}\left[\left|A_{1}\right|^{2}+2 \operatorname{Re}\left(A_{1}^{*} A_{2}\right)\right],
$$

where $\mathcal{C}$ is a normalization factor and $E=E_{1}+E_{2}$ is the kinetic energy of the two electrons.

Since the amplitude $A_{1}$ is linear in the polarization vector $\mathbf{e}$ of the field $\mathbf{F}(t)$, it can be expressed in terms of scalar products of the unit vectors $\hat{\mathbf{p}}_{1}$ and $\hat{\mathbf{p}}_{2}$ of the electron momenta $\mathbf{p}_{1}$ and $\mathbf{p}_{2}$, with the vector $\mathbf{e}_{\phi}$ :

$$
A_{1}=f_{1}(\xi)\left(\hat{\mathbf{p}}_{1} \cdot \mathbf{e}_{\phi}\right)+f_{2}(\xi)\left(\hat{\mathbf{p}}_{2} \cdot \mathbf{e}_{\phi}\right),
$$

where $\xi$ denotes the parameters $p_{1}, p_{2}, \cos \theta=\left(\hat{\mathbf{p}}_{1} \cdot \hat{\mathbf{p}}_{2}\right)$. In $L S$ coupling, the coordinate part of the wave function of the ${ }^{1} S_{0}$ initial state is symmetric under particle exchange; hence the amplitudes are symmetric under the exchange of photoelectron momenta $\mathbf{p}_{1} \leftrightarrow \mathbf{p}_{2}$, i.e., $f_{1}\left(p_{2}, p_{1}, \cos \theta\right)=f_{2}\left(p_{1}, p_{2}, \cos \theta\right)$. Introducing the symmetric $\left(f_{g}\right)$ and antisymmetric $\left(f_{u}\right)$ functions $f_{g / u}=f_{1} \pm f_{2}$ and the notations $\hat{\mathbf{p}}_{ \pm}=\left(\hat{\mathbf{p}}_{1} \pm \hat{\mathbf{p}}_{2}\right) / 2$, the parametrization (3) for $A_{1}$ in terms of $f_{g}(\xi)$ and $f_{u}(\xi)$ becomes

$$
A_{1}=e^{-i \phi}\left[f_{g}(\xi)\left(\mathbf{e} \cdot \hat{\mathbf{p}}_{+}\right)+f_{u}(\xi)\left(\mathbf{e} \cdot \hat{\mathbf{p}}_{-}\right)\right],
$$

where $f_{u}=0$ for equal energy sharing (EES), $p_{1}=p_{2}$.

The amplitude $A_{2}$ may be parametrized similarly and its CEP dependence may be expressed explicitly as

$$
A_{2}=e^{-2 i \phi} B\left(\xi ; \hat{\mathbf{p}}_{+}, \hat{\mathbf{p}}_{-}, \mathbf{e}, \mathbf{e}\right)+B^{\prime}\left(\xi ; \hat{\mathbf{p}}_{+}, \hat{\mathbf{p}}_{-}, \mathbf{e}, \mathbf{e}^{*}\right),
$$

where $B$ describes absorption of two photons and $B^{\prime}$ describes two-photon processes involving emission of one photon and absorption of another. Both $B$ and $B^{\prime}$ are superpositions of 
four $\xi$-dependent dynamical parameters multiplied by scalar products of the vectors involved:

$$
\begin{aligned}
B= & h(\xi)(\mathbf{e} \cdot \mathbf{e})+h_{+}(\xi)\left(\hat{\mathbf{p}}_{+} \cdot \mathbf{e}\right)^{2}+h_{-}(\xi)\left(\hat{\mathbf{p}}_{-} \cdot \mathbf{e}\right)^{2} \\
+ & h_{0}(\xi)\left(\hat{\mathbf{p}}_{+} \cdot \mathbf{e}\right)\left(\hat{\mathbf{p}}_{-} \cdot \mathbf{e}\right), \\
B^{\prime}= & h^{\prime}(\xi)+h_{+}^{\prime}(\xi)\left|\hat{\mathbf{p}}_{+} \cdot \mathbf{e}\right|^{2}+h_{-}^{\prime}(\xi)\left|\hat{\mathbf{p}}_{-} \cdot \mathbf{e}\right|^{2} \\
& +h_{0}^{\prime}(\xi) \operatorname{Re}\left\{\left(\hat{\mathbf{p}}_{+} \cdot \mathbf{e}\right)\left(\hat{\mathbf{p}}_{-} \cdot \mathbf{e}^{*}\right)\right\},
\end{aligned}
$$

where $\mathbf{e} \cdot \mathbf{e}=\ell$ is the degree of linear polarization of $\mathbf{F}(t)$.

In accordance with the general considerations discussed above, the AD of DPI electrons produced by an intense few-cycle pulse is asymmetric. The asymmetry is defined by $\Delta \mathcal{W}=\mathcal{W}\left(\mathbf{p}_{1}, \mathbf{p}_{2}, \mathbf{e}\right)-\mathcal{W}\left(\mathbf{p}_{1}, \mathbf{p}_{2},-\mathbf{e}\right) \equiv \mathcal{W}_{+\mathbf{e}}-\mathcal{W}_{-\mathbf{e}}$, or, alternatively, by Eq. (1). The explicit form for the asymmetry (1) in the PT regime follows upon substituting Eqs. (4) and (5) into Eq. (2) to obtain

$$
\begin{aligned}
\Delta \mathcal{W}\left(\mathbf{p}_{1}, \mathbf{p}_{2}, \mathbf{e}\right)= & 4 \mathcal{C} \operatorname{Re}\left\{\left[f_{g}^{*}(\xi)\left(\hat{\mathbf{p}}_{+} \cdot \mathbf{e}^{*}\right)+f_{u}^{*}(\xi)\left(\hat{\mathbf{p}}_{-} \cdot \mathbf{e}^{*}\right)\right]\right. \\
& \left.\times\left[e^{-i \phi} B+e^{i \phi} B^{\prime}\right]\right\},
\end{aligned}
$$

where we have used the invariance of $\left|A_{1}\right|^{2}$ under the transformation $\mathbf{e} \rightarrow-\mathbf{e}$. Hence the CEP dependence of $\Delta \mathcal{W}$ is given by a linear superposition of $\sin \phi$ and $\cos \phi$ terms [whose coefficients can be found by comparison with Eq. (8)]. According to PT, its dependence on the peak field intensity $I$ (relative to a reference value $\left.I_{r}\right)$ is $\Delta \mathcal{W} \propto\left(I / I_{r}\right)^{3 / 2}$. The asymmetry (8) is nonzero for both EES (when $f_{u}=0$ ) and unequal energy sharing (UES) and vanishes only for back-toback emission $\left(\hat{\mathbf{p}}_{+}=\mathbf{0}\right)$ in the former case (and, of course, after phase averaging). The selection rules applicable to the amplitude $A_{1}$ in Eq. (4) are clearly exhibited in Eq. (8).

\section{NUMERICAL RESULTS FOR THE CASE OF A LINEARLY POLARIZED PULSE}

For a general polarization e, solution of the two-electron TDSE is a numerically difficult, six-dimensional problem. We thus focus on the case of linear polarization $\mathbf{e}=\mathbf{e}^{*} \equiv \hat{\mathbf{z}}$, which reduces the numerical task to a five-dimensional problem. For simplicity of presentation, our numerical results are for the "in-plane" geometry, i.e., the vectors $\mathbf{p}_{1}, \mathbf{p}_{2}$, and $\hat{\mathbf{z}}$ lie in the same plane and one of the electron momenta $\mathbf{p}_{1}, \mathbf{p}_{2}$ is collinear with $\hat{\mathbf{z}}$. Our calculations include four values of $L$ : $0 \leqslant L \leqslant 3$, so that the rather small third-order PT amplitude effects are included implicitly. We assume a pulse envelope $F_{0}(t)=F_{0} \cos ^{2}(\pi t / T)$ with $-T / 2 \leqslant t \leqslant T / 2$, where $T \equiv$ $n_{c}(2 \pi / \omega)$ is the total pulse duration and $n_{c}=3$ optical cycles. The temporal full width at half maximum of the pulse intensity profile is $0.364 T=1.1$ cycles, which is comparable to those of the single-cycle pulses achieved experimentally $[15,16]$. The spectral width $\Delta \omega \simeq 1.44 \omega / n_{c}$ [41] of the pulse intensity profile is $31.2 \mathrm{eV}$ for $\omega=65 \mathrm{eV}(T=190.9$ as) and $40.8 \mathrm{eV}$ for $\omega=85 \mathrm{eV}$ ( $T=146 \mathrm{as})$. Significant asymmetries occur only at excess energies $E$ at which the PT amplitudes $\left|A_{1}\right|$ and $\left|A_{2}\right|$ have comparable magnitudes. Based on the pulse bandwidth and the DPI threshold energy $(\approx 79 \mathrm{eV})$, we estimate the energies at which these amplitudes interfere to be in the vicinity of $E \approx 30 \mathrm{eV}$ for $\omega=85 \mathrm{eV}$ and $E \approx 4 \mathrm{eV}$ for $\omega=65 \mathrm{eV}$. The peak pulse intensity is 1 or $2 \mathrm{PW} / \mathrm{cm}^{2}$.
The TDP for DPI is calculated by projecting the continuum part $\Phi_{C}(\phi)$ of the two-electron wavepacket solution of the full-dimensional, two-electron TDSE [47,48] (treating the electron-electron interaction exactly) onto field-free, uncorrelated symmetrized products of two Coulomb functions for $Z=$ 2. Our numerical method for obtaining $\Phi_{C}(\phi)$ is described in Ref. [13], which treats single ionization of $\mathrm{He}$ for a pulse carrier frequency $\omega=36 \mathrm{eV}$. We bypass the necessity of including correlation in the field-free double-continuum states by waiting $\approx 20$ a.u. after the end of the pulse before projecting our fully correlated two-electron wave packet onto the field-free states [35-37,41,44,45,49]. All results were checked for numerical convergence as in Refs. [35-37,44,45].

\section{A. Influence of the pulse carrier frequency on DPI electron angular distribution asymmetries}

The TDPs for $\omega=85 \mathrm{eV}$ and $65 \mathrm{eV}(E=29.6 \mathrm{eV}$ and $4 \mathrm{eV}), I=2 \mathrm{PW} / \mathrm{cm}^{2}$, and a CEP of $\phi=\pi / 2$ are displayed in Figs. 1 and 2. For each energy $E$, both EES and UES cases are shown. In each case, the electron with energy $E_{1}$ is detected on the symmetry axis, alternately along $+\hat{\mathbf{z}}$ or $-\hat{\mathbf{z}}$ (i.e., $\theta_{1}=0$ or $\pi)$. The $\mathrm{AD}$ of the electron with energy $E_{2}$ is then displayed as a function of $\theta_{2} \equiv \theta$, where $0 \leqslant \theta \leqslant \pi$. For the EES cases shown in Figs. 1(a) and 2(a), $A_{1}=0$ for back-to-back emission
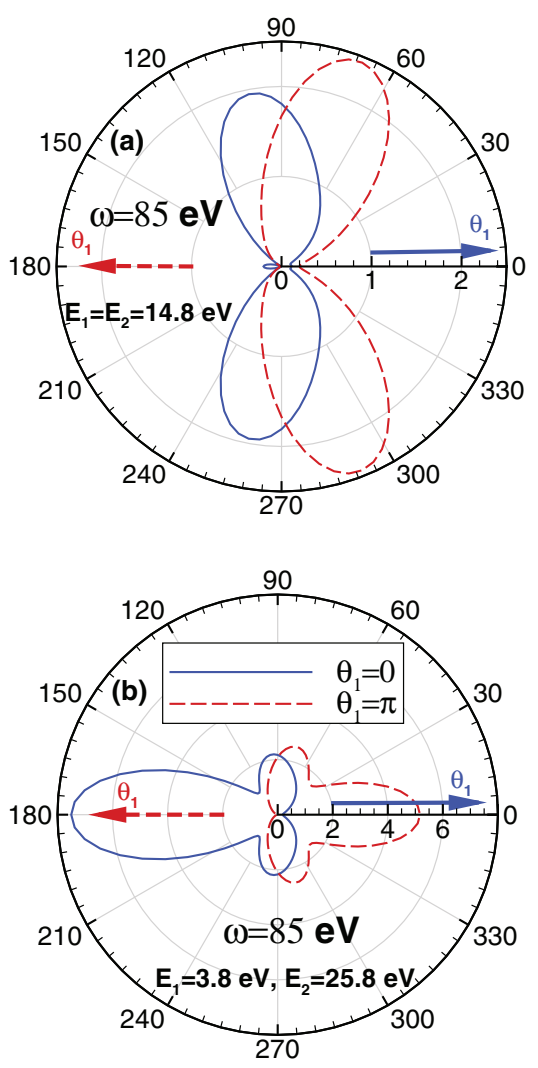

FIG. 1. (Color online) The TDP $d^{3} W / d E d \Omega_{\hat{\mathbf{p}}_{1}} d \Omega_{\hat{\mathbf{p}}_{2}}$ (in units of $10^{-7}$ a.u.) for DPI of $\mathrm{He}$ by a three-cycle attosecond pulse having a peak pulse intensity $I=2 \mathrm{PW} / \mathrm{cm}^{2}$, a CEP $\phi=\pi / 2$, and carrier frequency $\omega=85 \mathrm{eV}$ for (a) EES and (b) UES. The electron energies $E_{1}$ and $E_{2}$ are specified in each panel. Solid (blue) curve, $\theta_{1}=0$ [solid (blue) arrow], $0 \leqslant \theta_{2} \leqslant \pi$; dashed (red) curve, $\theta_{1}=\pi$ [dashed (red) arrow], $0 \leqslant \theta_{2} \leqslant \pi$. 

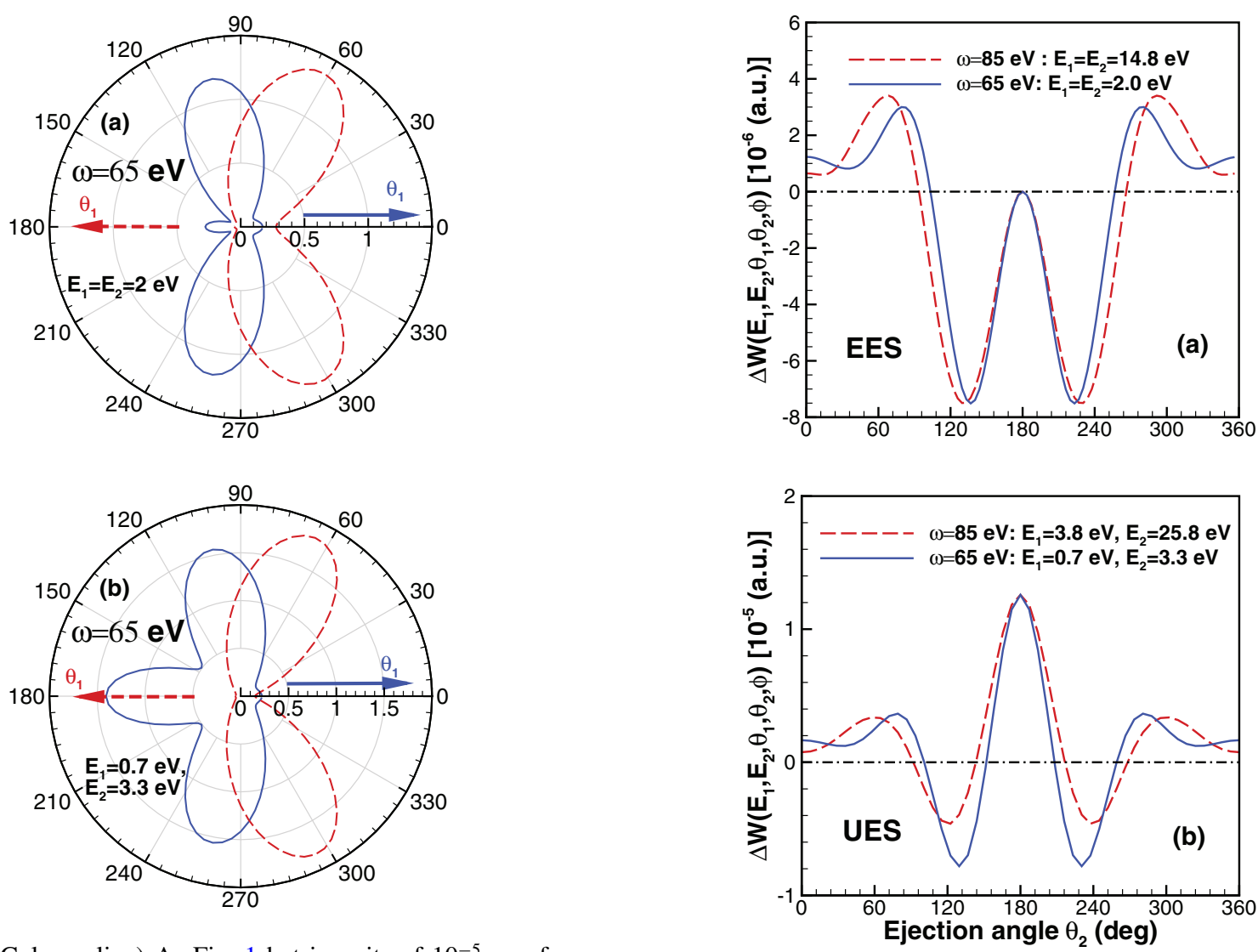

FIG. 2. (Color online) As Fig. 1 but in units of $10^{-5}$ a.u. for a carrier frequency $\omega=65 \mathrm{eV}$ for (a) EES and (b) UES.

$(\theta=\pi)$, since both $\hat{\mathbf{p}}_{+}$and $f_{u}$ in Eq. (4) are zero. In this case, Eq. (2) must be replaced by $\mathcal{W}\left(\mathbf{p}_{1},-\mathbf{p}_{1}, \hat{\mathbf{z}}\right)=\mathcal{C}\left|A_{2}\right|^{2}$; it is thus a direct measure of the second-order TDP. Since $\left|A_{2}\right|^{2}$ is invariant to $\hat{\mathbf{z}} \rightarrow-\hat{\mathbf{z}}$, the on-axis values of the two TDPs for $\theta_{2}=0$ and $\pi$ are equal in both Figs. 1(a) and 2(a). The two large lobes in these figures originate predominantly from interference between the amplitudes $f_{g}$ and $f_{u}$ in $A_{1}$ [3]; however, their angular positions relative to $\theta_{1}$ differ for $\theta_{1}=0$ and $\pi$, in contrast to the case of a long pulse [3]. In the UES cases shown in Figs. 1(b) and 2(b), $A_{1} \neq 0$ in the back-to-back configuration. For $\omega=85 \mathrm{eV}$ [cf. Fig. 1(b)] one sees two large, asymmetric on-axis lobes along $\theta_{2}=0$ and $\pi$. In contrast, for $\omega=65 \mathrm{eV}$ [cf. Fig. 2(b)], which has a small excess energy $E=4 \mathrm{eV}$, one of the corresponding lobes is significant, but the other is very small.

In Fig. 3 we plot as a function of $\theta_{2}$ the difference between the pair of TDP curves in each panel of Figs. 1 and 2, i.e., the asymmetry $\Delta \mathcal{W}\left(\mathbf{p}_{1}, \mathbf{p}_{2}, \hat{\mathbf{z}}\right)$, which for the case of linear polarization is convenient to parametrize as

$$
\Delta \mathcal{W}\left(\mathbf{p}_{1}, \mathbf{p}_{2}, \hat{\mathbf{z}}\right)=|K|\left(I / I_{r}\right)^{3 / 2} \cos \left(\phi+\Theta_{K}\right),
$$

where $K(\xi, \hat{\mathbf{z}}) \equiv|K| \exp \left(i \Theta_{K}\right)$ is a CEP- and intensityindependent dynamical parameter, defined by comparison with Eq. (8). One sees that the shape of $\Delta \mathcal{W}$ is insensitive to $\omega$, but that its magnitude is highly sensitive to $\omega$. This latter sensitivity stems from the proportionality of $\Delta \mathcal{W}$ to the interference term $2 \operatorname{Re}\left(A_{1}^{*} A_{2}\right)$ in Eq. (2). The results in Fig. 3 show that although $\omega=65 \mathrm{eV}$ is below the $79 \mathrm{eV}$ threshold for DPI, the bandwidth of the pulse is large enough that overlap of the

FIG. 3. (Color online) Asymmetry $\Delta \mathcal{W}$ for DPI of He by a threecycle pulse with peak intensity $2 \mathrm{PW} / \mathrm{cm}^{2}$ and CEP $\phi=\pi / 2$ for two carrier frequencies $\omega$. Both EES (a) and UES (b) cases are shown. Results for $\omega=85 \mathrm{eV}$ are increased by factors of 71 and 54 in (a) and (b), respectively.

first- and second-order amplitudes is largest at that frequency. For EES, $\Delta \mathcal{W}$ vanishes for $\theta_{2}=\pi$ owing to the node in the $A_{1}$ amplitude in the back-to-back configuration. In contrast, for UES, $\Delta \mathcal{W}$ takes its maximum value at this angle. The normalized asymmetry $R \equiv \Delta \mathcal{W} /\left(\mathcal{W}_{+\hat{\mathbf{z}}}+\mathcal{W}_{-\hat{\mathbf{z}}}\right)$ at this angle is $18 \%$ for $\omega=85 \mathrm{eV}$ and $80 \%$ for $\omega=65 \mathrm{eV}$. For the EES case at $\theta_{2}=136.8^{\circ}, R \approx 52 \%$ for 65 and $85 \mathrm{eV}$.

\section{B. CEP-induced electron angular distribution asymmetries in DPI of He by a few-cycle XUV pulse}

The dependence of the TDP $d^{3} W / d E d \Omega_{\hat{\mathbf{p}}_{1}} d \Omega_{\hat{\mathbf{p}}_{2}}$ on the CEP $\phi$ is obtained explicitly upon substituting Eqs. (4) and (5) into Eq. (2). The results for three CEPs are shown for the EES case in Figs. 4(a)-4(c) and for the UES case in Figs. 5(a)-5(c). The sensitive dependence on the CEP shown in these figures stems from the interference of the first- and second-order PT amplitudes [cf. Eq. (2)]. This is confirmed in Figs. 4(d) and 5(d) in which only the first-order term $\left|A_{1}\right|^{2}$ is taken into account in Eq. (2). Numerically, the results in Figs. 4(d) and 5(d) are obtained by projecting (long after the pulse) the two-electron wave packet onto the ${ }^{1} P^{o}$ final state of two Coulomb functions. From Eq. (4) one sees that $\left|A_{1}\right|^{2}$ has no dependence on the CEP and also the ADs for $\theta_{1}=0^{\circ}$ and $\theta_{1}=180^{\circ}$ are then mirror images of one another, unlike the CEP-dependent cases in the first three panels of Figs. 4 and 5. The effects of the interference 

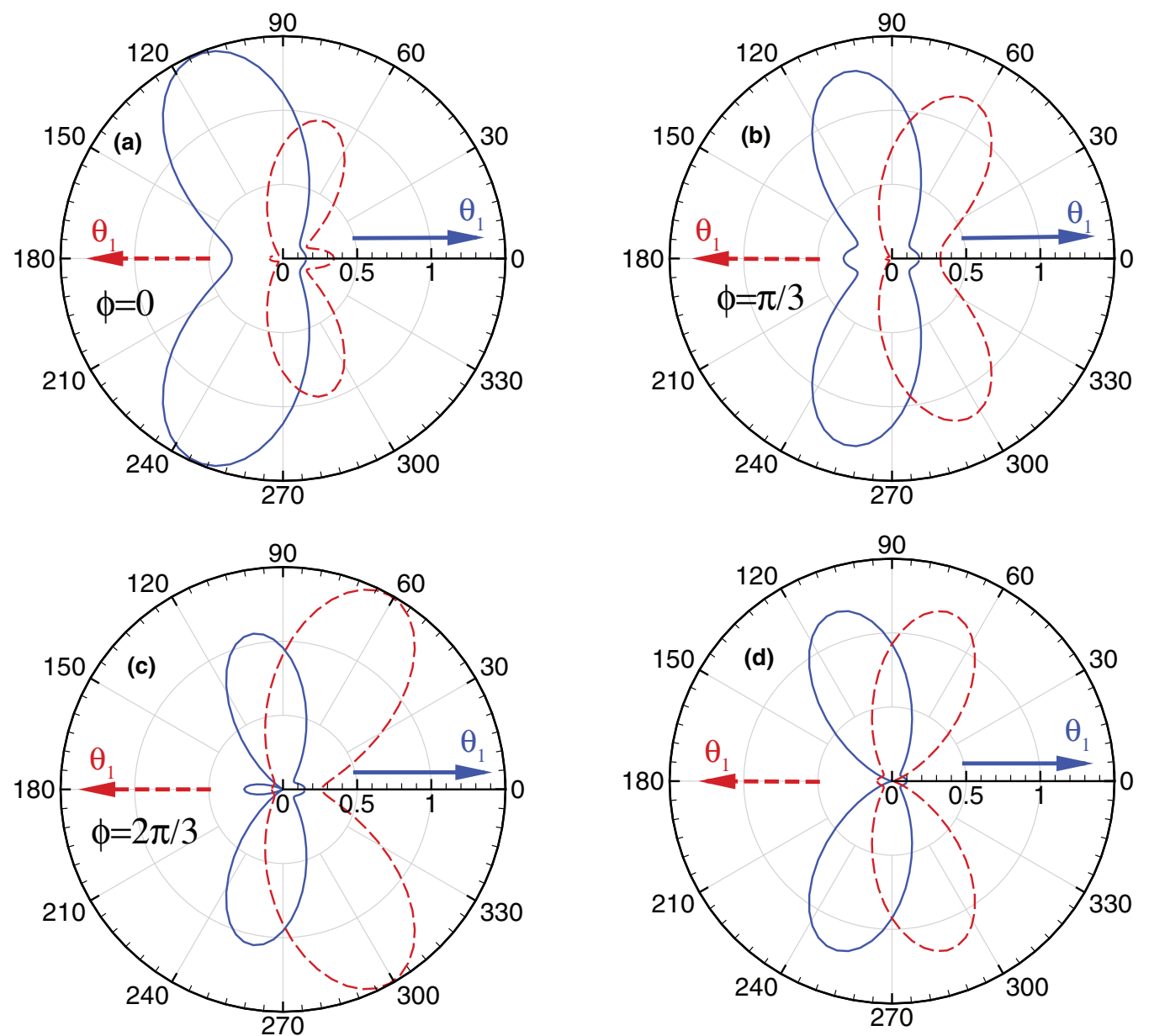

FIG. 4. (Color online) The TDP $d^{3} W / d E d \Omega_{\hat{\mathrm{p}}_{1}} d \Omega_{\hat{\mathrm{p}}_{2}}$ (in units of $10^{-5}$ a.u.) for DPI of He by a three-cycle XUV pulse with carrier frequency $\omega=65 \mathrm{eV}$ and peak pulse intensity of $I=2 \mathrm{PW} / \mathrm{cm}^{2}$ for three CEPs: (a) $\phi=0$, (b) $\phi=\pi / 3$, and (c) $\phi=2 \pi / 3$. In (d) we give for comparison the TDP obtained by including only the first-order PT amplitude. All results are for the EES case with $E_{1}=E_{2}=4 \mathrm{eV}$ and the color coding is the same as in Fig. 1.

term $2 \operatorname{Re}\left(A_{1}^{*} A_{2}\right)$ on the TDP [cf. Eq. (2)] are more pronounced in the UES cases shown in Fig. 5.

Qualitatively and regardless of energy sharing, in each panel one can identify the two twin lobes of the $\mathrm{AD}$ originating from the $\left|A_{1}\right|^{2}$ term in Eq. (2), which is modified by the addition of the CEP-dependent interference term $2 \operatorname{Re}\left(A_{1}^{*} A_{2}\right)$. Both the magnitude and the angular shift of these twin lobes depend sensitively on the CEP. The on-axis lobe at $\theta=180^{\circ}$ in each panel originates mainly from the second-order PT amplitude $A_{2}$. [In the EES cases, since $A_{1}$ vanishes at $\theta=180^{\circ}$, the on-axis lobes seen in Figs. 4(a)-4(c) are due to $\left|A_{2}\right|^{2}$, which although not included in Eq. (2) is included implicitly in our numerical calculations.] The lobe at $\theta=180^{\circ}$ in each of the EES and UES cases attains its maximum magnitude at $\phi \approx$ $\pi / 6$. For the EES case, the lobe has its minimum magnitude at $\phi \approx \pi / 2$ [cf. Fig. 2(a)].

When changing $\phi \rightarrow \phi \pm \pi$ or $\hat{\mathbf{z}} \rightarrow-\hat{\mathbf{z}}$ and comparing $\mathcal{W}_{-\hat{\mathbf{z}}}\left(\mathbf{p}_{1}, \mathbf{p}_{2}, \phi\right)$ with $\mathcal{W}_{+\hat{\mathbf{z}}}\left(\mathbf{p}_{1}, \mathbf{p}_{2}, \phi\right)$ [see Figs. 4(a)-4(c) and $5(\mathrm{a})-5(\mathrm{c})]$ one finds a significant difference between the electron ADs, leading thus to large CEP-induced asymmetries in $\Delta \mathcal{W}$ [cf. Eq. (8)], whose CEP dependence is shown in Fig. 6 for three different geometries. As is seen in Fig. 6, the asymmetries $\Delta \mathcal{W}$ at $\phi=0$ and $\phi=\pi$ differ only in their sign.
This originates from the fact that the replacement $\phi \rightarrow \phi \pm \pi$ is equivalent to the inversion of the photon polarization vector $\mathbf{e} \rightarrow-\mathbf{e}$, which, in turn, results in the inversion of the sign of $\Delta \mathcal{W}$.

Confirmation of the validity of PT for DPI of He by a few-cycle XUV pulse with the parameters we have employed is obtained by fitting the PT parametrization of $\Delta \mathcal{W}$ in Eq. (9) to our full-dimensional, two-electron TDSE results. The results of fits for three cases are shown in Fig. 6. The fitted PT curves are in excellent agreement with the TDSE results. The dynamical parameters $K \equiv|K| \exp \left(i \Theta_{K}\right)$ for those three configurations and one other are given in Table I for two peak pulse intensities. One sees that the magnitude $|K|$ is invariant to a doubling of the peak intensity to within $\leqslant 5 \%$, while the phase $\Theta_{K}$ is invariant to within $\approx 3^{\circ}$.

\section{CEP dependence of electron angular distributions in the orthogonal geometry}

In the orthogonal geometry (for any energy sharing) the two ionized electrons are ejected perpendicular to the plane of the laser pulse polarization ellipse (or to the direction of the polarization vector for a linearly polarized pulse field). 

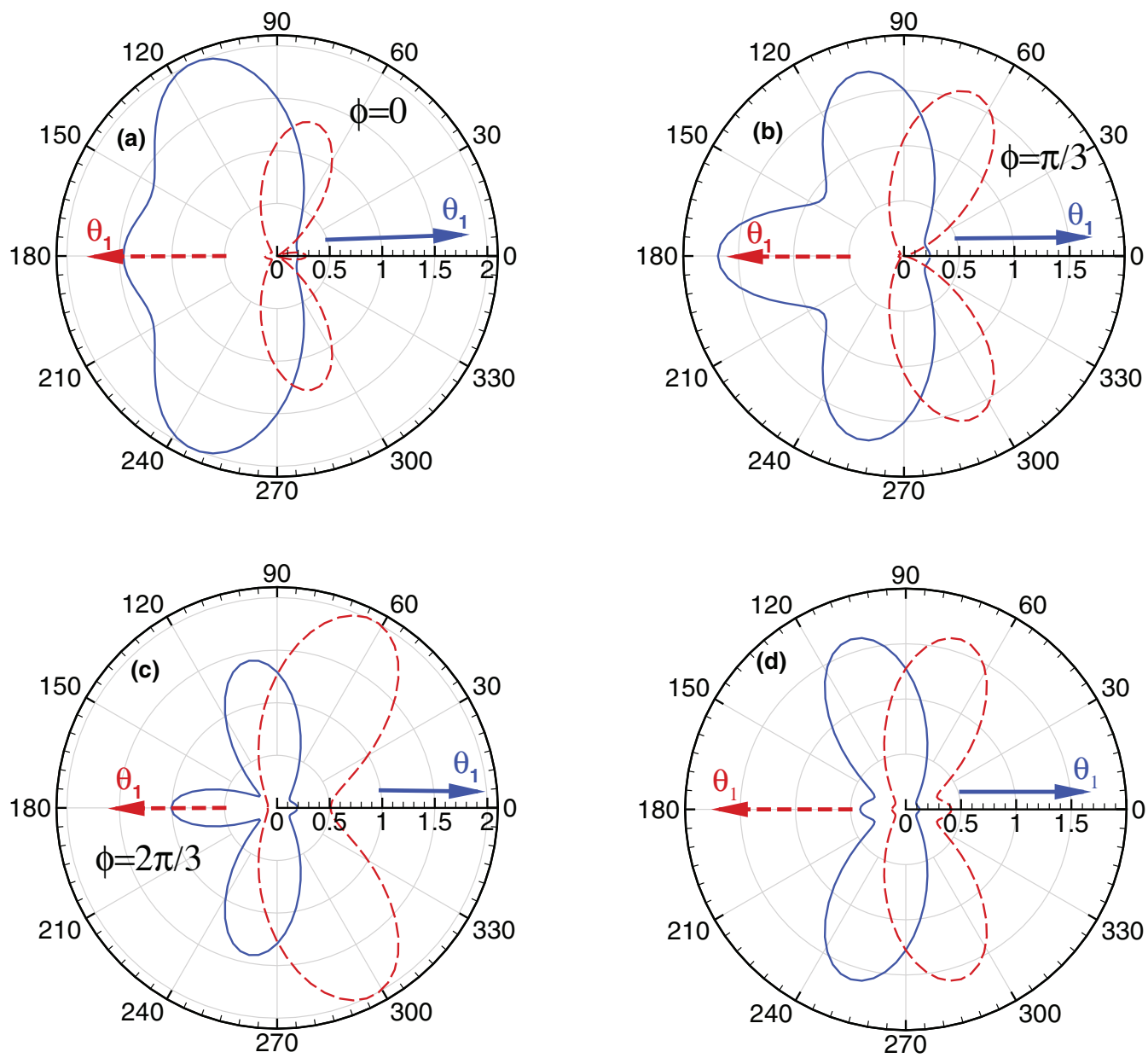

FIG. 5. (Color online) As in Fig. 4 but for the UES case with $E_{1}=0.7 \mathrm{eV}$ and $E_{2}=3.3 \mathrm{eV}$.

For this geometry, as one can see from Eq. (4), the first-order PT DPI amplitude vanishes since $\hat{\mathbf{p}}_{+} \cdot \mathbf{e}=0$ and $\hat{\mathbf{p}}_{-} \cdot \mathbf{e}=0$.

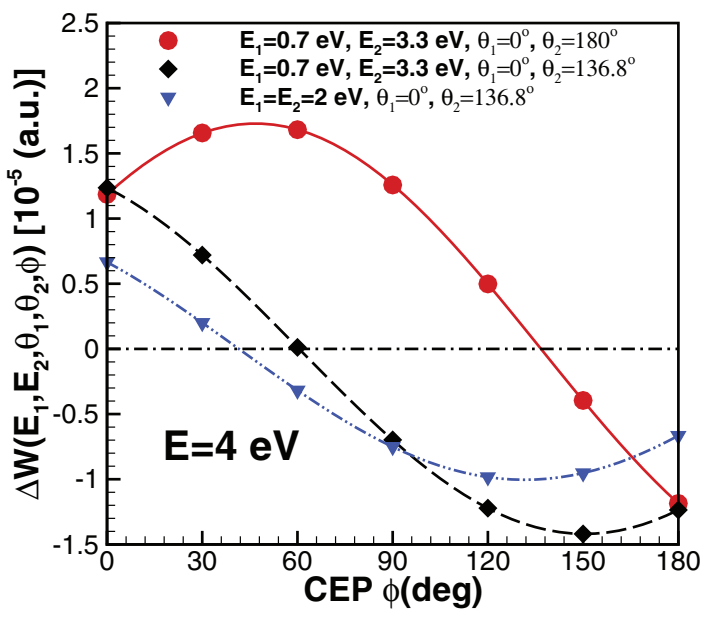

FIG. 6. (Color online) CEP dependence of the asymmetry $\Delta \mathcal{W}$ for DPI of He by a three-cycle pulse with $\omega=65 \mathrm{eV}$, and $I=$ $2 \mathrm{PW} / \mathrm{cm}^{2}$. Three geometries with $E=4 \mathrm{eV}$ are shown, including both EES and UES cases, as specified in the figure legend. The symbols give our TDSE results. The lines are fits of the TDSE results to the PT parametrization (9).
Hence, the DPI probability is given by the absolute square of the second-order PT amplitude $A_{2}$, i.e., by $\mathcal{C}|A|^{2}$. From Eq. (5) one sees that the DPI probability is sensitive to the CEP of the pulse. This geometry thus provides a means to directly measure the CEP dependence of the second-order PT amplitude. For a given $\omega$, the $\mathrm{CEP}$ dependence has the parametrization:

$$
\mathcal{C}\left|A_{2}\right|^{2}=\alpha_{\omega}(I)+\beta_{\omega}(I) \cos (2 \phi)+\gamma_{\omega}(I) \sin (2 \phi),
$$

where $\alpha_{\omega}(I), \beta_{\omega}(I)$, and $\gamma_{\omega}(I)$ are CEP-independent, but intensity-dependent, dynamical parameters, defined by comparison with Eq. (5). (The intensity dependence is proportional

TABLE I. Dynamical parameters $|K|\left(10^{-5}\right.$ a.u. $)$ and $\Theta_{K}$ for $\omega=65 \mathrm{eV}$ obtained by fitting the PT parametrization in Eq. (9) to our TDSE results for $\Delta \mathcal{W}$ (with $I_{1}=1 \mathrm{PW} \mathrm{cm}^{-2}$ and $I_{2}=I_{r} \equiv$ 2 PW cm ${ }^{-2}$ ). EES cases: $\theta_{2}=$ (a) $136.8^{\circ}$ and (b) $133.2^{\circ}$. UES cases: $\theta_{2}=$ (c) $180^{\circ}$ and (d) $136.8^{\circ}$.

\begin{tabular}{lccccc}
\hline \hline $\operatorname{EES}(\mathrm{eV})$ & $K$ & (a) $I_{1}$ & (a) $I_{2}$ & (b) $I_{1}$ & (b) $I_{2}$ \\
\hline$E_{1}, E_{2}$ & $|K|:$ & 0.96 & 1.00 & 0.96 & 1.00 \\
$2.0,2.0$ & $\Theta_{K}:$ & $45.9^{\circ}$ & $48.5^{\circ}$ & $44.6^{\circ}$ & $47.4^{\circ}$ \\
$\mathrm{UES}(\mathrm{eV})$ & $K$ & (c) $I_{1}$ & (c) $I_{2}$ & (d) $I_{1}$ & (d) $I_{2}$ \\
\hline$E_{1}, E_{2}$ & $|K|:$ & 1.81 & 1.73 & 1.41 & 1.42 \\
$0.7,3.3$ & $\Theta_{K}:$ & $316^{\circ}$ & $313^{\circ}$ & $26.2^{\circ}$ & $29.5^{\circ}$ \\
\hline \hline
\end{tabular}



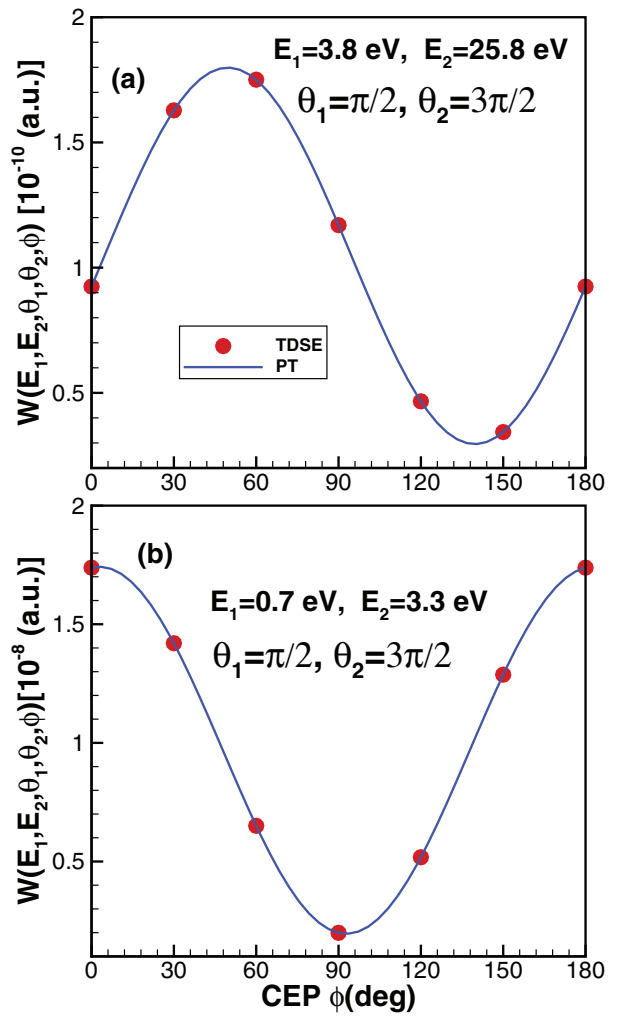

FIG. 7. (Color online) CEP dependence of the DPI TDP for an orthogonal, back-to-back geometry $\left(\theta_{1}=\pi / 2, \theta_{2}=3 \pi / 2\right)$ for two UES cases (indicated in each panel) and two carrier frequencies: (a) $\omega=85 \mathrm{eV}$ and (b) $\omega=65 \mathrm{eV}$. The three-cycle XUV pulse has a peak intensity of $2 \times 10^{15} \mathrm{~W} / \mathrm{cm}^{2}$. The red filled circles are the TDSE results and the blue lines are fits of the TDSE results to the PT parametrization in Eq. (10).

to $I^{2}$, as our numerical results, not shown, confirm.) For the case of linear polarization, it is convenient to parametrize the TDP in Eq. (10) as

$$
\mathcal{C}\left|A_{2}\right|^{2}=\alpha_{\omega}(I)\left[1+\tilde{\beta}_{\omega} \cos \left(2 \phi+\Theta_{\omega}\right)\right],
$$

where $\tilde{\beta}_{\omega}$ and $\Theta_{\omega}$ are defined by comparing with Eq. (10).

In Fig. 7 we present the CEP dependence of the DPI TDP for two UES cases involving back-to-back electron emission in the plane orthogonal to the direction of linear polarization of an XUV pulse. In Fig. 7(a) the UES results are for $E_{1}=3.8 \mathrm{eV}$ and $E_{2}=25.8 \mathrm{eV}$, corresponding to an XUV pulse carrier frequency of $\omega=85 \mathrm{eV}$, whereas in Fig. 7(b) the UES results are for $E_{1}=0.7 \mathrm{eV}$ and $E_{2}=3.3 \mathrm{eV}$ and a carrier frequency of $\omega=65 \mathrm{eV}$. Note that the results for $\omega=85 \mathrm{eV}$ are about two orders of magnitude smaller than those for $\omega=65 \mathrm{eV}$ and both results are significantly suppressed (by about three orders of magnitude) compared to the case of the "in-plane" geometry. In each case the TDSE numerical results (indicated by the filled circles) are fitted to the parametrization in Eq. (11), giving excellent agreement between the numerical and parametrized CEP dependence. The extracted dynamical parameters obtained from the fits for these two configurations for $I=2 \mathrm{PW} \mathrm{cm}^{-2}$ are respectively $\alpha_{85}=1.05 \times 10^{-10}$ a.u., $\tilde{\beta}_{85}=0.72, \Theta_{85}=260.6^{\circ}$; and $\alpha_{65}=$ $96.9 \times 10^{-10}$ a.u., $\tilde{\beta}_{65}=0.80, \Theta_{65}=-5.68^{\circ}$. One sees that in contrast to the phase shift $\Theta_{\omega}$, the amplitude $\tilde{\beta}_{\omega}$ of the CEP-dependent term in Eq. (11) is only slightly sensitive to the carrier frequency.

\section{SUMMARY AND CONCLUSIONS}

In summary, we have presented a parametrization of twoelectron ADs for DPI of He by an intense, few-cycle XUV pulse based on PT. Owing to the broad bandwidth of the pulse, first- and second-order PT amplitudes are nonzero over an overlapping range of electron kinetic energies $E$. Their interference produces asymmetric electron ADs that can be controlled by the CEP of the pulse. For most geometries, the ADs are proportional to the first-order DPI amplitude, so that selection rules for various experimental configurations known from single-photon DPI are applicable also to DPI by a few-cycle pulse. For linear polarization of the pulse, our PT parametrization is in excellent agreement with results of solutions of the full-dimensional, two-electron TDSE, validating the PT approach. These numerical results show that the normalized asymmetry in DPI significantly exceeds that for single ionization of He for $I=1-2 \mathrm{PW} / \mathrm{cm}^{2}[12,13]$ and are comparable to that for single ionization plus excitation of He to $\mathrm{He}^{+}(2 s, 2 p)$ [14]. We have also investigated the CEP dependence of the AD for the special case of orthogonal geometry for which the first-order amplitude vanishes, providing a means to directly investigate the CEP dependence of the second-order PT amplitude. In general, our analytic results here indicate for any geometry how to control ionized electrons spatially by means of the XUV pulse parameters $(\omega, \mathrm{CEP}$, and polarization). Other geometries and pulse polarizations will be analyzed elsewhere. A similar analysis for DPI of He involving overlap between the second- and the third-order transition amplitudes leading to asymmetric $\operatorname{ADs}\left[\Delta \mathcal{W}\left(\mathbf{p}_{1}, \mathbf{p}_{2}, \mathbf{e}\right) \propto\right.$ $\left(I / I_{r}\right)^{5 / 2}$ for the peak field intensity $I$ relative to a reference value $I_{r}$ ] can be readily performed. Note finally that if and when intense, few-cycle attosecond pulses become available, experiments for DPI of He are likely to be among the first performed owing to the availability of reaction-microscope techniques for electron momentum spectroscopy [50].

\section{ACKNOWLEDGMENTS}

We gratefully acknowledge Suxing $\mathrm{Hu}$, for providing us with his FEDVR-TDCC code for solving the two-electron TDSE, and Bernard Piraux, for helpful discussions. This work is supported in part by DOE, Office of Science, Division of Chemical Sciences, Geosciences, and Biosciences, Grant No. DE-FG03-96ER14646 and by RFBR Grant No. 13-02-00420. Our computations used the Sandhills facility, Holland Computing Center, University of Nebraska and the NSF TeraGrid resources, NICS Kraken Supercomputer, Grant No. TG-PHY-120003.
[1] J. S. Briggs and V. Schmidt, J. Phys. B 33, R1 (2000).

[2] F. Maulbetsch and J. S. Briggs, J. Phys. B 28, 551 (1995).
[3] P. Lablanquie, J. Mazeau, L. Andric, P. Selles, and A. Huetz, Phys. Rev. Lett. 74, 2192 (1995). 
[4] N. L. Manakov, S. I. Marmo, and A. V. Meremianin, J. Phys. B 29, 2711 (1996).

[5] G. G. Paulus, F. Grasbon, H. Walther, P. Villorese, M. Nisoli, S. Stagira, E. Priori, and S. De Silvestri, Nature (London) 414, 182 (2001).

[6] G. G. Paulus, F. Lindner, H. Walther, A. Baltuška, E. Goulielmakis, M. Lezius, and F. Krausz, Phys. Rev. Lett. 91, 253004 (2003).

[7] D. B. Milošević, G. G. Paulus, D. Bauer, and W. Becker, J. Phys. B 39, R203 (2006).

[8] M. V. Frolov, D. V. Knyazeva, N. L. Manakov, A. M. Popov, O. V. Tikhonova, E. A. Volkova, M.-H. Xu, L.-Y. Peng, L.-W. Pi, and A. F. Starace, Phys. Rev. Lett. 108, 213002 (2012).

[9] X. Liu et al., Phys. Rev. Lett. 93, 263001 (2004).

[10] A. Staudte et al., Phys. Rev. Lett. 99, 263002 (2007).

[11] E. A. Pronin, A. F. Starace, M. V. Frolov, and N. L. Manakov, Phys. Rev. A 80, 063403 (2009).

[12] L.-Y. Peng, E. A. Pronin, and A. F. Starace, New J. Phys. 10, 025030 (2008).

[13] J. M. Ngoko Djiokap, S. X. Hu, W.-C. Jiang, L.-Y. Peng, and A. F. Starace, New J. Phys. 14, 095010 (2012).

[14] J. M. Djiokap, S. X. Hu, W. C. Jiang, L. Y. Peng, and A. F. Starace, Phys. Rev. A 88, 011401(R) (2013).

[15] G. Sansone et al., Science 314, 443 (2006).

[16] E. Goulielmakis et al., Science 320, 1614 (2008).

[17] S. Gilbertson, Y. Wu, S. D. Khan, M. Chini, K. Zhao, X. Feng, and Z. Chang, Phys. Rev. A 81, 043810 (2010).

[18] E. J. Takahashi, P. Lan, O. D. Mücke, Y. Nabekawa, and K. Midorikawa, Phys. Rev. Lett. 104, 233901 (2010).

[19] P. Lan, E. J. Takahashi, and K. Midorikawa, Phys. Rev. A 82, 053413 (2010).

[20] T. Popmintchev, M.-C. Chen, P. Arpin, M. M. Murnane, and H. C. Kapteyn, Nat. Photonics 4, 822 (2010).

[21] F. Ferrari, F. Calegari, M. Lucchini, C. Vozzi, S. Stagira, G. Sansone, and M. Nisoli, Nat. Photonics 4, 875 (2010).

[22] P. Lan, E. J. Takahashi, and K. Midorikawa, Phys. Rev. A 83, 063839 (2011).

[23] P. Tzallas, E. Skantzakis, L. A. A. Nikolopoulos, G. D. Tsakiris, and D. Charalambidis, Nat. Phys. 7, 781 (2011).

[24] J. M. Mikhailova, M. V. Fedorov, N. Karpowicz, P. Gibbon, V. T. Platonenko, A. M. Zheltikov, and F. Krausz, Phys. Rev. Lett. 109, 245005 (2012).

[25] H. Hasegawa, E. J. Takahashi, Y. Nabekawa, K. L. Ishikawa, and K. Midorikawa, Phys. Rev. A 71, 023407 (2005).

[26] A. A. Sorokin, M. Wellhöfer, S. V. Bobashev, K. Tiedtke, and M. Richter, Phys. Rev. A 75, 051402(R) (2007).

[27] A. Rudenko et al., Phys. Rev. Lett. 101, 073003 (2008).
[28] M. Kurka et al., New J. Phys. 12, 073035 (2010).

[29] A. Rudenko et al., J. Phys. B 43, 194004 (2010).

[30] S. Laulan, H. Bachau, B. Piraux, J. Bauer, and G. Lagmago Kamta, J. Mod. Opt. 50, 353 (2003).

[31] B. Piraux, J. Bauer, S. Laulan, and H. Bachau, Eur. Phys. J. D 26, 7 (2003)

[32] S. Laulan and H. Bachau, Phys. Rev. A 68, 013409 (2003).

[33] E. Foumouo, G. L. Kamta, G. Edah, and B. Piraux, Phys. Rev. A 74, 063409 (2006).

[34] E. Foumouo, Ph. Antoine, H. Bachau, and B. Piraux, New J. Phys. 10, 025017 (2008).

[35] J. Feist, S. Nagele, R. Pazourek, E. Persson, B. I. Schneider, L. A. Collins, and J. Burgdörfer, Phys. Rev. A 77, 043420 (2008).

[36] X. Guan, K. Bartschat, and B. I. Schneider, Phys. Rev. A 77, 043421 (2008).

[37] J. Feist, S. Nagele, R. Pazourek, E. Persson, B. I. Schneider, L. A. Collins, and J. Burgdörfer, Phys. Rev. Lett. 103, 063002 (2009).

[38] J. Feist, R. Pazourek, S. Nagele, E. Persson, B. I. Schneider, L. A. Collins, and J. Burgdörfer, J. Phys.: Conf. Ser. 194, 012010 (2009).

[39] A. Palacios, T. N. Rescigno, and C. W. McCurdy, Phys. Rev. Lett. 103, 253001 (2009).

[40] Z. Zhang, L.-Y. Peng, Q. Gong, and T. Morishita, Opt. Express 18, 8976 (2010).

[41] E. Foumouo, A. Hamido, Ph. Antoine, B. Piraux, H. Bachau, and R. Shakeshaft, J. Phys. B 43, 091001 (2010).

[42] A. Palacios, D. A. Horner, T. N. Rescigno, and C. W. MncCurdy, J. Phys. B 43, 194003 (2010).

[43] R. Pazourek, J. Feist, S. Nagele, E. Persson, B. I. Schneider, L. A. Collins, and J. Burgdörfer, Phys. Rev. A 83, 053418 (2011).

[44] Z. Zhang, L.-Y. Peng, M.-H. Xu, A. F. Starace, T. Morishita, and Q. Gong, Phys. Rev. A 84, 043409 (2011).

[45] L. Argenti, R. Pazourek, J. Feist, S. Nagele, M. Liertzer, E. Persson, J. Burgdörfer, and E. Lindroth, Phys. Rev. A 87, 053405 (2013).

[46] W.-C. Jiang, L.-Y. Peng, W.-H. Xiong, and Q. Gong, Phys. Rev. A 88, 023410 (2013).

[47] B. I. Schneider, L. A. Collins, and S. X. Hu, Phys. Rev. E 73, 036708 (2006).

[48] S. X. Hu, Phys. Rev. E 81, 056705 (2010).

[49] L. B. Madsen, L. A. A. Nikolopoulos, T. K. Kjeldsen, and J. Fernández, Phys. Rev. A 76, 063407 (2007).

[50] J. Ullrich, R. Moshammer, A. Dorn, R. Dörner, L. Ph. H. Schmidt, and H. Schmidt-Böcking, Rep. Prog. Phys. 66, 1463 (2003). 